\title{
TOWARD A NEW GENERATION OF WORLD CLIMATE RESEARCH AND COMPUTING FACILITIES
}

\author{
by J. Shukla, T. N. Palmer, R. Hagedorn, B. Hoskins, \\ J. Kinter, J. Marotzke, M. Miller, and J. Slingo
}

\begin{abstract}
To accelerate progress in understanding and predicting regional climate change, national climate research facilities must be enhanced and dedicated multi-national facilities should be established.
\end{abstract}

W eather and climate are undisputedly major factors for the well-being and development of society, impacting all scales from individual lives to global economies (Sachs 2008). Societies have flourished by adapting to and taking advantage of current climate conditions. However, this relationship between climate and society is fragile and volatile: during the past 25 years, weather-related disasters have caused more than 600,000 fatalities and

AFFILIATIONS: SHUKLA AND KINTER-George Mason University (GMU), Fairfax, Virginia, and COLA/IGES, Calverton, Maryland; Hagedorn AND Miller-European Centre for Medium-Range Weather Forecasts (ECMWF), Reading, United Kingdom; HoskINs - Imperial College London, London, United Kingdom, and University of Reading, Reading, United Kingdom; MAROtZKE-Max Planck Institute for Meteorology, Hamburg, Germany; PALMERECMWF, Reading, United Kingdom, and Oxford University, Clarendon Laboratory, Oxford, United Kingdom; SLINGO-Met Office, Exeter, United Kingdom, and Department of Meteorology, University of Reading, Reading, United Kingdom CORRESPONDING AUTHOR: Prof. Jagadish Shukla, GMU/IGES, 404I Powder Mill Road, Suite 302, Calverton, MD 20705-3106 E-mail: shukla@cola.iges.org

The abstract for this article can be found in this issue, following the table of contents.

DOI:10.1175/2010BAMS2900.1

In final form 18 December 2009

(C) 2010 American Meteorological Society
$\$ 1.3$ trillion (U.S. dollars) of economic losses. This paper is part of an ensemble of papers proposing an international multidisciplinary prediction initiative (Shapiro et. al. 2010).

Considering the increasing frequency of extreme weather and climate events (Alley et al. 2007) together with our enhanced vulnerability (WMO 2006) to weather and climate hazards caused by rapid economic and population growth, mortality and economic losses will continue to rise. As the Stern report has emphasized (Stern 2007), climate change is a trilliondollar problem: inaction will be many times costlier than cutting greenhouse gas emissions, which itself could cost the world economy as much as $1 \%$ of its gross domestic product (GDP).

The Intergovernmental Panel on Climate Change (IPCC) has alerted society to the risk the world faces from climate change, and governments are formulating climate-change-related policies. However, formulating cost-effective and responsible mitigation and adaptation strategies raises questions about specifics of climate change. How far can greenhouse gas concentrations rise before dangerous climate changes are inevitable? How big an investment does society need to adapt to already inevitable climate changes? How will climate change regionally, not just in terms of temperature but of other key variables such as precipitation and storminess? For example, what is needed to ensure that people in regions at risk of increased drought will 
have adequate water, while those in regions at risk of increased precipitation are sufficiently protected against flooding? Questions such as "Is New Orleans sustainable?" or "When should we begin planning to accommodate the hundreds of millions of climate refugees from low-lying areas who will be left homeless if ice sheets begin to disintegrate?" will have to be answered. What if society is unable to substantially reduce $\mathrm{CO}_{2}$ emissions? What are the consequences of radical measures to offset global warming?

Answering such questions will require detailed, quantitative predictions of climate change for the coming century on both global and regional scales, and with sufficient detail to provide statistics on hazardous weather events. It will be imperative that the predictions have a high level of reliability given their influence on decision making. While current IPCC models show robust levels of consistency in some aspects of climate change, large disagreements among these models indicate considerable uncertainty about the magnitude of future climate change, especially on the regional scale. Furthermore, the inability of current climate models to describe accurately key modes of variability of climate indicates common model deficiencies. This makes the long-term prediction of regional climate even more uncertain.

At the World Modelling Summit for Climate Prediction (Shukla et. al. 2009), scientists (http:// wcrp.wmo.int) declared that they currently lack the appropriate tools (models and computers) to make the step change in climate prediction required to give fully trustworthy answers to the questions posed by society. The summit reflected scientists' near universal agreement that resolution of climate models must improve to accurately represent key regional processes in the atmosphere and in the oceans. Constrained by insufficient climate-dedicated computing power, contemporary climate models resort to highly truncated representations of the mathematical laws of physics. Scientists must simplify key physical processes.

Both from simple theory and by inspection of diagnostics of model-generated energy spectra, the scales reasonably described by a numerical model of the atmosphere or ocean are many times larger than the nominal grid length. This means a typical climate model (with $150-\mathrm{km}$ grids in the atmosphere and $100-\mathrm{km}$ grids in the ocean) cannot represent many subsynoptic-scale systems at all and only poorly represent many smaller baroclinic features. These models underestimate the number of storms actually observed and poorly simulate the statistics of midlatitude blocking (Jung et al. 2006). Recent results from a fully coupled climate model with higher resolution in both the atmosphere $(90 \mathrm{~km})$ and ocean $\left(1 / 3^{\circ}\right)$ show significant benefits from capturing the coherent coupling on these finer scales (Shaffrey et al. 2009). Studies with much higher resolution in NWP have shown that this can both improve the description of important structures within synoptic weather systems and provide opportunities to capture highly energetic mesoscale systems (Jung and Rhines 2007).

It is often said that high-resolution regional models, widely used to provide climate impact assessments at the regional and local level, can be used to provide information including, for example, the changing frequency and intensity of hurricanes. This is a questionable concept in the context of climate change (WCRP 2007). Regional downscaling cannot capture the global teleconnections of regional variations and cannot improve this aspect of regional prediction. For example, the frequency of Atlantic hurricanes is strongly influenced by Pacific Ocean temperatures. It can be argued that only a global highresolution climate model can estimate the changes in the frequency and intensity of hurricanes, and indeed other high-impact weather events, in a changing climate. We believe it is unacceptable to make major decisions about the habitability of hurricane-affected coastal areas using information from climate models that cannot resolve hurricanes, especially because scientific knowledge and technology now allow better.

The requirement to increase resolution of climate models is based on considerable experience with weather and short-term climate prediction where predictive skill has steadily improved during the past 30 years as models use higher resolution and better representations of physical processes. The experience of Japanese research groups in simulating global weather using a powerful supercomputer, the Earth Simulator, and horizontal resolution of 3.5-10 $\mathrm{km}$ has established the scientific basis for very high-resolution models (Miura et al. 2007; Oouchi et al. 2009) and begun the process of probing the benefits of increased resolution. In the last 20 years or so operational weather centers have made several significant spatial resolution upgrades. Each change to higher resolution improves representation of basic components such as orography and land-sea definition, synoptic and subsynoptic systems, and weather features such as fronts, cloud- and rainbands, and jets. Resolution also improves assimilation of observations, and these together have contributed significantly to the long-term improvements in objective forecast skill at the major NWP centers. Further refinements in resolution can be expected to continue to improve forecast skill and simulation fidelity, although this is not universally accepted. In particular, the possibility 
exists that there is an irreducible level of uncertainty below which further refinements in model resolution cannot reach. The overwhelming opinion of those present at the summit is that that limit has not nearly been reached.

In addition to the merits of running climate models at a resolution comparable with that of NWP models, the continual confrontation of an NWP model with observations can provide important constraints when the same model is used for much longer-time-scale climate predictions. The seamless nature of the prediction problem was highlighted in the WCRP Strategy for 2005-15 (WCRP 2005), and an ability to apply the insights and constraints of NWP to climate is one of the key motivations for the development of so-called seamless prediction systems, as discussed by Palmer and Webster (1995) and Brunet et al. (2010).

Short-range (limited area) forecast models give encouraging results using grid lengths of close to $1 \mathrm{~km}$, without parameterizing deep convection. For example, the Met Office currently runs an operational limited-area model at $1.5 \mathrm{~km}$, which is beginning to deliver substantial improvements in skill in forecasting extreme rainfall events, especially when the synoptic forcing is strong (Lean et al. 2008). Since a discrete model can reasonably resolve features more than 5 times the grid length, individual deep convective clouds are still far from resolved. Nevertheless, these models improve resolution of mesoscale structures such as downdraft outflows. Much more realistic kinetic energy spectra are also captured with grids of $10 \mathrm{~km}$ or less (Terasaki et al. 2009).

However, higher resolution alone is not sufficient to improve climate simulation; we will need new physical parameterizations. For example, extensive research needs to be done to ensure that once the grid resolution is high enough $(\sim 1 \mathrm{~km})$ to resolve convective cloud systems, the rapid growth of the uncertainties in these unpredictable features does not overwhelm the predictable largest-scale flow; in fact, at that resolution, computational factors challenge the whole algorithmic design of a global climate model.

Furthermore, the requirement for better physical climate representation-of deep convective cloud systems, energetic eddies in the oceans, etc.-is only one of several reasons climate prediction modeling must have substantially more powerful and dedicated computing and research infrastructure:

- Representation of biogeochemical processes in climate models (to predict, for instance, concentration of radiatively active gases) is crude, and we do not know how much Earth system processes depend on the resolution of the physical climate system (Nobre et. al. 2010).

- Prediction uncertainty-a key variable - can be estimated by making an ensemble of forecasts with varying initial conditions, model equations, and other input fields such as greenhouse gas concentrations, but this is currently not feasible with models of sufficient resolution or complexity.

- We must run climate models over paleoclimatic epochs to assess, for example, whether they can simulate past glacial cycles and abrupt climate changes; currently there are insufficient computer resources to run even current resolution climate models over periods much longer than a few centuries.

- The ingestion of Earth observations into climate models requires the development of data assimilation capability, an exceptionally complex computational process currently impossible in most climate institutes because of the lack of computer resources.

Together with the problem of insufficient resolution, the above considerations collectively argue for the very substantial augmentation of computer power and research effort that the World Modeling Summit argued would be necessary to accelerate progress in climate modeling and fulfill society's need and expectations for reliable, quantitative predictions of regional climate change.

WHAT IS NECESSARY TO FULFILL SOCIETY'S EXPECTATIONS? It is somewhat ironic that the science community uses climate models with inadequate spatial resolution because of the lack of powerful computers and a shortage of qualified scientists teaming up to build models. The scientific basis and knowledge for building such models, and the evidence that higher-resolution and better representation of physical processes improves predictions, have been available for more than 20 years. Because current computational infrastructures are funded through national resources, no single modeling center in the world has been able to acquire the required supercomputing resources and the critical mass of scientists to build and run climate models with cloud-system-resolving atmosphere, eddy-resolving oceans, and landscape-resolving land surfaces to investigate what is really needed scientifically to provide confident global and regional predictions over the next century. The operational NWP centers have been able to increase the spatial resolution of global weather forecast models from about $100 \mathrm{~km}$ in 1990 to about $20 \mathrm{~km}$ today because 
they do not have the additional constraints of having to include more complexity and having to run models for multidecades. Both weather and climate research has seen about a 1000 -fold increase over the past 25 years in the sustained speed of computers from about 1 gigaflop (1 gigaflop $=10^{9}$ floating point operations per second) to about 1 teraflop ( 1 teraflop $=10^{12}$ floating point operations per second). For example, the computer at the European Centre for MediumRange Weather Forecasts (ECMWF), with a sustained speed of about 2 teraflops, currently can produce 10 -day weather forecasts in about 20 minutes with a global atmosphere model of about $20-\mathrm{km}$ resolution. If, for argument's sake, we were to run the ECMWF weather forecast model as a climate model coupled to an equally complex and computationally demanding global ocean model, both with about 4-km resolution, achieving a century of simulation each month, the sustained speed of the computers would have to increase another 1000 -fold to about 2 petaflops $\left(1\right.$ petaflop $=10^{15}$ floating point operations per second). To achieve the same performance, the Earth Simulator in Japan (Satoh et al. 2008), would need to increase its speed by a factor of 250 . This does not take into consideration the additional computing power needed for more complexity (biogeochemistry, etc.) to advance from physical climate system models to true Earth system models or for larger ensembles.

This may be possible within three years. The manufacturers of high-performance computers predict that by then peak computing performance will be about 20 petaflops (e.g., http://www-03.ibm.com/press/ us/en/pressrelease/26599.wss). Within the five years following the introduction of multipetaflop machines, peak speeds might reach 200 petaflops and 1 exaflop ( 1 exaflop $=10^{18}$ floating point operations per second) by the end of the decade, giving sustained performance of up to 20 petaflops on typical fluid dynamical codes. Given this potential, large domain limited-area models with 1-km grids and a systematic study of the sensitivity of global coupled models to both horizontal and vertical resolution are likely to be the best ways to improve simulations through better (possibly stochastic-dynamic) parameterizations, improved physical understanding of the coupled system, and better appreciation of methodological shortcomings.

We probably will not meet the "grand challenge" of running global climate models at $1-\mathrm{km}$ resolution in the next 10 years, even if our investigation of resolution suggested that this was optimal, but this should not prevent extensive experimentation on basic weather-climate interactions at cloud-systempermitting resolution.
Using very high-performance computing power efficiently requires adequate infrastructure and human resources. First of all, current climate model software is not designed for the massively parallel architecture of multipetaflop machines. This implies that major recoding will be needed to fully exploit future computers. A sufficient number of computing specialists and climate scientists have to be gathered and motivated to work together to achieve this challenging goal.

Second, the data volumes produced by climate models will increase by orders of magnitudes. This will pose enormous technical challenges of archiving, interrogating, analyzing, and visualizing the data. Again, efficient and optimum utilization of the outputs will require novel solutions and thus the collaboration of many dedicated technical and scientific experts. Therefore, it is imperative that the facilities proposed here comprise both computing and human resources. Only the two components together will ensure the success of this new endeavor.

\section{HOW CAN WE REALIZE THE NECESSARY \\ FACILITIES? Climate models have traditionally} been developed by individual government institutes and university departments. However, in the last few decades it has become a well-established tenet that many of the most scientifically and technically challenging problems can only be tackled by infrastructure unaffordable at the national level. Countries have come together to fund high-energy particle accelerators to probe matter on the smallest scales and powerful telescopes to probe the universe on the largest scales. Indeed, the Consultative Group on International Agricultural Research (CGIAR) is credited with launching the green revolution leading to sustainable food security and poverty reduction in the world (Evenson and Gollin 2003). Recently, several countries announced the creation of a single jointly funded laboratory to create sustainable nuclear fusion (www.iter.org/index.htm).

The success of international investment in scientific and technical infrastructure is also apparent in weather and seasonal climate prediction, as illustrated by the European Centre for Medium-Range Weather Forecasts and the International Research Institute for Climate and Society. The climate science community needs the sort of international infrastructure that physicists, astronomers, and others now take for granted. Considering the need for creative competition, we propose the creation of a small number (at least three) of international high-performance computing facilities dedicated entirely to climate research to facilitate the creation of the next-generation 
global climate models with the capabilities we have described. Machines with the capability to support this groundbreaking research will not be otherwise available to national centers in the coming decade. Concerted international funding would stimulate the computing industry to accelerate the development and delivery of the sort of machine required.

Climate change is a global problem and therefore it is reasonable that the solution to reliable predictions also lies at the global level. The cost of a single facility (on the order of $\$ 1$ billion in workforce and computers over 5 years) could be affordable if shared among the nations of the world with contributions in proportion to national GDP.

At present, the huge quantity of space-based observations cost tens of billions of dollars annually. The spatial resolution of satellite data $(1-10 \mathrm{~km})$ is so much finer than model resolution $(50-200 \mathrm{~km})$ and the research and computing infrastructure is so inadequate that only a small fraction of space-based observations is being assimilated in forecast models. A supercomputing facility with sustained petaflop computing capability that a critical mass of scientists can access will also accelerate progress in new methods of data assimilation that will be needed to initialize decadal predictions.

It now appears likely that the multipetaflop machines in the United States, Europe, and Japan will be funded at the national level, but each will serve multiple scientific applications. Because there are many demands on very high-performance computing (nanotechnology, biology, astrophysics, high-energy physics, and so on), climate applications will only receive a small share of time on such national-level computing infrastructure. Moreover, the machine architecture may not necessarily be optimal for climate simulation. Sharing time on such computers would certainly not provide the best possible opportunities for accelerating research and model development to provide better climate predictions. Establishing a small number of dedicated computing facilities at the international level will enable that revolution so that, in the future, predictions can be made using models whose realism is commensurate with the need to guide policy on mitigation and on adaptation strategies. Funding for such multinational facilities should not and cannot compete with the funding for national centers, which will still be needed to provide the predictions and services that their country requires. In fact, funding for the national centers should also be enhanced so that they can make best use of the developments in the science and modeling enabled by the multinational facilities. As the products delivered by the national centers and services improve, we anticipate that the national centers and services will grow in value and funding. The need for improvements in global and regional climate predictions will remain for at least several decades and such multinational facilities should not be subject to year-to-year funding uncertainties. Given the humanitarian implications, private corporations, wealthy individuals, and foundations should be approached to create an endowment fund to guarantee sustained funding.

The return on investments in enhancing climate modeling by creating major international research and computing facilities is expected to be quite high. Such facilities would almost certainly help national weather services to improve the day-to-day weather forecasts of extreme, high-impact weather events and provide invaluable information to agencies that manage weather-related disasters. The new generation of models will yield improved statistics of daily weather and, therefore, better predictions of regional climate variations on seasonal time scales. Even modest improvements in the prediction of seasonal and interannual variations will provide huge socioeconomic benefits through applications in agriculture, water resources, health, and energy sectors.

Based on these estimates, we propose that governments, the computing industry, and the science community work together toward the common goal of establishing a small number of international computing facilities with computer capability of 20 petaflops in near terms, 200 petaflops within the subsequent five years, and 1 exaflop by the end of the next decade. This two-step approach would enable us to achieve the more immediate goal of running global climate models for multidecadal climate predictions and IPCC projections at a resolution of about $10 \mathrm{~km}$. Parallel to that, these facilities will be used to address the many research questions related to the grand challenge of running global climate models at a much higher resolution of about $1 \mathrm{~km}$. These are, of course, current goals. By the time individual weather services might have acquired such computers for routine weather prediction, the international facilities will be producing climate predictions using computers of a much higher capability.

\section{REVOLUTIONIZING CLIMATE MODELING} AND PREDICTION. Since current climate systems models are not able to provide predictions with adequate accuracy and detail, climate prediction needs to be revolutionized to be able to fulfill society's expectations. To accelerate progress in understanding the mechanisms of climate variations 
and predicting climate change with reliable estimates of uncertainties, the existing national facilities should be enhanced, and in addition a small number of multinational research and computing facilities dedicated to climate research, with resources and capabilities beyond those of a single national effort, should be established. We recommend the creation of a small number (at least three) of highly connected multinational facilities with computer capability for each facility of at least 20 petaflops in the near term, 200 petaflops within five years, and 1 exaflop by the end of the next decade.

Soon the societal demand for policy-relevant climate predictions will be so great that the most advanced technology and the best available talent must be brought to bear to address this great challenge. The time to begin that process is now!

\section{REFERENCES}

Alley, R. B., and Coauthors 2007: Summary for policymakers. Climate Change 2007: The Physical Science Basis, S. Solomon et al., Eds., Cambridge University Press, 1-18.

Brunet, G., and Coauthors, 2010: Collaboration of the weather and climate communities to advance subseasonal to seasonal prediction. Bull. Amer. Meteor. Soc., 91, 1397-1406.

Evenson, R. E., and D. Gollin, 2003: Assessing the impact of the Green Revolution, 1960 to 2000. Science, 300, 758-762.

Hermann, A., L. Belloni, U. Mersits, D. Pestre, and, J. Krige, 1987: History of CERN, I: Volume I-Launching the European Organization for Nuclear Research. North-Holland, $622 \mathrm{pp}$.

Jung, T., and P. B. Rhines, 2007: Greenland's pressure drag and the Atlantic storm track. J. Atmos. Sci., 64, 4004-4030.

—, S. K. Gulev, I. Rudeva, and V. Soloviov, 2006: Sensitivity of extratropical cyclone characteristics to horizontal resolution in the ECMWF model. Quart. J. Roy. Meteor. Soc., 132, 1839-1857.

Lean, H., P. A. Clark, M. Dixon, N. M. Roberts, A. Fitch, R. Forbes, and C. Halliwell, 2008: Characteristics of high-resolution versions of the Met Office Unified Model for forecasting convection over the United Kingdom. Mon Wea Rev., 136, 3408-3424.

Miura, H., M. Satoh, T. Nasuno, A. T. Noda, and K. Oouchi, 2007: A Madden-Julian oscillation event simulated using a global cloud-resolving model. Science, 318, 1763-1765.

Nobre, C., G. Brasseur, M. Shapiro, M. Lahsen, G. Brunet, A. Busalacchi, K. Hibbard, and K. Noone,
2010: Addressing the complexity of the Earth system. Bull. Amer. Meteor. Soc., 91, 1389-1396.

Oouchi, K., A. T. Noda, M. Satoh, B. Wang, S.-P. Xie, H. Takahashi, and T. Yasunari, 2009: Asian summer monsoon simulated by a global cloudsystem-resolving model: Diurnal to intra-seasonal variability. Geophys. Res. Lett., 36, L11815, doi:10.1029/2009GL038271.

Palmer, T. N., and P. J. Webster, 1995: Towards a unified approach to climate and weather prediction. Global Change: Proc. First Demetra Meeting, Chianciano Terme, Italy, European Commission, EUR 15158 EN, 265-280 pp.

Rodwell, M., and T. N. Palmer, 2007: Using numerical weather prediction to assess climate models. Quart. J. Roy. Meteor. Soc., 133, 129-146.

Sachs, J. D., 2008: Common Wealth: Economics for a Crowded Planet. Penguin Press, 400 pp.

Satoh, M., T. Nasuno, H. Miura, H. Tomita, S. Iga, and Y. Takayabu, 2008: Precipitation statistics comparison between global cloud resolving simulation with NICAM and TRMM PR data. High Resolution Numerical Modelling of the Atmosphere and Ocean, W. Ohfuchi and K. Hamilton, Eds., Springer, 99-112.

Shaffrey, L., and Coauthors, 2009: UK-HiGEM: The new U.K. High-Resolution Global Environment ModelModel description and basic evaluation. J. Climate, 22, 1861-1896.

Shapiro, M. A., and Coauthors, 2010: An Earth-system prediction initiative for the twenty-first century. Bull Amer. Meteor. Soc., 91, 1377-1388.

Shukla, J., R. Hagedorn, B. Hoskins, J. Kinter, J. Marotzke, M. Miller, T. Palmer, and J. Slingo, 2009: Revolution in climate prediction is both necessary and possible: A declaration at the World Modelling Summit for Climate Prediction. Bull. Amer. Meteor. Soc., 90, 16-19.

Stern, N., 2007: The Economics of Climate Change: The Stern Review. Cambridge University Press, 692 pp.

Terasaki, K., H. L. Tanaka, and M. Satoh, 2009: Characteristics of the kinetic energy spectrum of NICAM model atmosphere. SOLA, 5, 180-183.

WCRP, 2005: The World Climate Research Programme Strategy 2005-15. WMO/TD-No. 1291, 59 pp.

- 2007: Report of the twenty-eighth session of the Joint Scientific Committee. WMO/TD 1395, 173-176.

WMO, cited 2006: Summary statement on tropical cyclones and climate change. World Meteorological Organization. [Available online at www.wmo.int/ pages/prog/arep/press_releases/2006/pdf/iwtc_summary.pdf.] 